\title{
A New DG Multiobjective Optimization Method Based on an Improved Evolutionary Algorithm
}

\author{
Wanxing Sheng, Ke-yan Liu, Yongmei Liu, Xiaoli Meng, and Xiaohui Song \\ Power Distribution Research Department, China Electric Power Research Institute, No. 15, Xiaoying East Road, \\ Qinghe, Haidian District, Beijing 100192, China
}

Correspondence should be addressed to Ke-yan Liu; liukeyan@epri.sgcc.com.cn

Received 16 January 2013; Revised 19 March 2013; Accepted 21 March 2013

Academic Editor: Ricardo Perera

Copyright (c) 2013 Wanxing Sheng et al. This is an open access article distributed under the Creative Commons Attribution License, which permits unrestricted use, distribution, and reproduction in any medium, provided the original work is properly cited.

\begin{abstract}
A distribution generation (DG) multiobjective optimization method based on an improved Pareto evolutionary algorithm is investigated in this paper. The improved Pareto evolutionary algorithm, which introduces a penalty factor in the objective function constraints, uses an adaptive crossover and a mutation operator in the evolutionary process and combines a simulated annealing iterative process. The proposed algorithm is utilized to the optimize DG injection models to maximize DG utilization while minimizing system loss and environmental pollution. A revised IEEE 33-bus system with multiple DG units was used to test the multiobjective optimization algorithm in a distribution power system. The proposed algorithm was implemented and compared with the strength Pareto evolutionary algorithm 2 (SPEA2), a particle swarm optimization (PSO) algorithm, and nondominated sorting genetic algorithm II (NGSA-II). The comparison of the results demonstrates the validity and practicality of utilizing DG units in terms of economic dispatch and optimal operation in a distribution power system.
\end{abstract}

\section{Introduction}

With the increasing demand for clean and renewable energy, the issue of distribution generation (DG) is drawing more attention worldwide. DG provides voltage support to largescale distribution power systems, which results in reliability improvements and reduction loss in the power grid. DG technology has become a hot research topic, given the increasing global concerns about environmental protection, energy conservation, and the increasing sophistication of wind power, photovoltaic power generation, and other renewable energy technologies. After DG is connected to a distribution network, the distribution network's structure, operation and control mode will tremendously change, and the distribution system automation and the demand-side management must consider the coordination between DG and distribution network control. Deciding the optimal DG output is a challenging research problem, especially considering the multiple optimal objectives associated with cases of multiple DG unit injections.

Traditionally, multi-objective DG optimization has been treated as a single-objective optimization problem using suitable weighting factors to form a weighted sum of single objectives. This approach has the disadvantage of finding only a single solution that does not express the tradeoffs with different weighting factors. Generating multiple solutions using this approach requires several runs with different factors, which leads to long running times [1, 2]. Recent studies have treated the objectives simultaneously and independently as a true multi-objective optimization problem. However, the optimization problem becomes more complicated due to such issues as continuity, local optima, linearization, and so forth. New optimization techniques, such as particle swarm optimization (PSO), different evolution (DE), and evolutionary programming (EP), have recently been introduced and applied in the field of power systems and with promising results [3-12]. In a recent study [3], a differential evolution approach was proposed to solve an optimal power flow problem with multiple objectives. The active power dispatch and reactive power dispatch were considered. A nonlinear constrained multi-objective optimization problem was formulated. A general overview of evolutionary multi-objective optimization was provided in [4], and the most representative algorithms were discussed. In [5], 
Pareto-based multi-objective evolutionary algorithms were discussed and evaluated. A nondominated sorting genetic algorithm (NSGA), a niched Pareto genetic algorithm, and a strength Pareto evolutionary algorithm (SPEA) were developed and applied to an environmental/economic electric power dispatch problem. A multi-objective formulation for sitting and sizing of DG units was proposed in [6]. The method involved searching for a compromise between the cost of network upgrades, cost of power losses, cost of energy not supplied, and cost of energy required by the served customers. A genetic algorithm was implemented to obtain a noninferior solution set. However, their method cannot guarantee a solution to be optimal solution. In [7], an improved swarm optimization (IPSO) method was presented to solve the multi-objective optimal power flow problem. The multiobjective optimal power flow considered the cost, loss, voltage stability and emission impacts as the objective functions. A fuzzy decision-based mechanism is used to select the best compromise Pareto set solution obtained by the proposed algorithm. In [8], a new penalty parameter-less constrainthandling scheme was employed to improve the performance of the evolutionary algorithm. The experiments in that paper revealed that PSO performs better in terms of solution quality and consistency, and DE performs better in terms of mean computation time. An improved Cai and Wang's method has been proposed to combine multi-objective optimization with differential evolution to address constrained optimization problems in [9]. The method provided a novel infeasible solution replacement mechanism for differential evolution in theory. In [10], a robust DE algorithm was proposed for the control of selective harmonic distortion and total harmonic distortion. A fuzzy optimization technique and DE optimization method are described.

The literature includes several DG output studies that examined multiple objectives and applied evolutionary optimization techniques. From the perspective of mathematical optimization, DG unit injection is a complex multiobjective optimization problem that presents a challenge to the optimization analysis of a distribution power system. The objectives include optimal energy consumption, the minimum power consumer's electricity purchasing cost, and the minimum power loss based on the constraints of power grid security and DG power output. Multi-objective economic/emission dispatch algorithms were investigated in $[11,12]$. In the optimization methods literature, the simulated annealing technique has been applied to optimize the proposed multi-objective model of DG planning [13]. The multiobjective problems were solved by converting the original model into an equivalent model through calibration of the weighted factors method. In [14], a multi-objective Tabu search- (TS-) based method was utilized to optimize a DG allocation problem. In that paper, the TS-based approach was provided to find the optimal Pareto set. Fuzzy optimization was also used to solve the multi-objective optimization of DG allocation in [15]. Voltage drop reduction, short circuit capacity augmentation, decrease operation cost, and system loss reduction were considered objectives for formulating fuzzy optimization.
In this paper, a DG multi-objective optimization method based on an improved evolutionary algorithm was investigated for a distribution power system. Adaptive crossover and a mutation operator were used in the evolutionary process, and simulated annealing was combined in the iterative process. A fuzzy clustering algorithm was applied to manage the size of the Pareto set. The rest of the paper is organized as follows. In Section 2, the formulation of the DG multiple-objective optimization for distribution management is presented. The Pareto-based algorithm and some basic concepts are introduced in Section 3. The improved Pareto evolution algorithm is described in Section 4. Section 5 provides the numerical results and comparison analysis with the proposed approach using the revised IEEE 33-bus system. The conclusion and future work are provided in Section 6.

\section{Problem Formulation}

2.1. Objective Functions. Three objectives are considered in the optimization model, which includes the fuel cost and the pollutant emission penalty, reducing consumer costs on electricity bills when DG units are injected into the distribution network and reducing transmission line losses. The first optimization objective is minimum energy consumption and a pollutant emission model, which is mainly based on government requirements. There will be more penalties if the system emits more pollutants and exhibits greater fuel consumption. The second objective is consumer related, where the consumer uses DG to maximize savings on their bills. The third objective is to lower system line losses, which is the demand objective of the power supply provider. The three objectives involve perspectives based on government requirements, consumer needs, and power supply enterprise needs, and the objectives can conflict. For example, when a consumer utilizes a micro gas turbine to maximize their savings on their energy bill, there is a subsequent increase in fuel cost and pollutant emission. In addition, the extra power from the micro gas turbine will increase or decrease the line losses, depending on the size and placement location of the micro gas turbine.

2.1.1. Fuel Cost and Pollutant Emission Minimization. The first objective is to minimize the fuel cost and the pollutant emission penalty, which reflects the impact of energy utilization on the environment. It can be expressed as follows:

$$
F_{1}(x)=\sum_{t=1}^{T}\left[C_{R}+C_{W}\right],
$$

where $C_{R}$ is the energy consumption cost and $C_{W}$ is the pollutant emission penalty. follows:

The fuel cost $C_{R}$ normally can be further expressed as

$$
C_{R}\left(P_{\mathrm{DG}}\right)=\sum_{i=1}^{N_{\mathrm{DG}}}\left(c_{i}+b_{i} P_{\mathrm{DG}_{i}}+a_{i} P_{\mathrm{DG}_{i}}^{2}\right) .
$$

Note that $c_{i}, b_{i}$, and $a_{i}$ are the quadratic cost coefficients of the $i$ th $\mathrm{DG}$, and $N_{\mathrm{DG}}$ is the number of distributed generators. 
$P_{\mathrm{DG}_{i}}$ is the real power output of the $i$ th generator. $P_{\mathrm{DG}}$ is the vector of real power outputs of generators and defined as follows:

$$
P_{\mathrm{DG}}=\left[P_{\mathrm{DG}_{1}}, P_{\mathrm{DG}_{2}}, \ldots, P_{\mathrm{DG}_{n}}\right]^{T} \text {. }
$$

The pollutant emission quantity can be obtained based on DG output. Then, based on the penalty standard, the environmental penalty for pollutant emission is calculated as follows:

$$
C_{W}=\sum_{j=1}^{P} Y_{j} D_{j}
$$

where $Y_{j}$ is pollutant $j$ 's emission quantity and $D_{j}$ is the penalty standard of pollutant $j$.

2.1.2. Maximization of Cost Savings Using DG. The second objective is to maximize the cost savings on electricity user bills when the DG is injected into the distribution network. The savings in electricity, which should have been purchased from the power supply enterprise, are the total power output of the DG units. Utilizing DG output and time-of-use (TOU) rate, consumer electricity purchasing costs could be reduced as follows:

$$
\begin{aligned}
\operatorname{maximize} \quad F_{2}(x)= & \sum_{t=T_{1}}^{T_{2}} C_{d_{1}} P_{\mathrm{DG}_{t}}+\sum_{t=0}^{T_{1}} C_{d_{2}} P_{\mathrm{DG}_{t}} \\
& +\sum_{t=T_{2}}^{24} C_{d_{2}} P_{\mathrm{DG}_{t}},
\end{aligned}
$$

where $C_{d_{1}}$ is peak price from $T_{1}$ to $T_{2}, C_{d_{2}}$ is off-peak price, and $P_{\mathrm{DG}_{t}}$ is the DG total power output at moment $t$.

2.1.3. Minimization of Line Losses. The third objective is to minimize the system line losses after DG injection into the distribution network. This objective can be expressed as follows:

$$
F_{3}(x)=P_{\text {loss }}=\sum_{i=0}^{m}\left(\frac{P[i]^{2}+Q[i]^{2}}{U[i]^{2}}\right) R[i],
$$

where $P[i]$ is the active power, $Q[i]$ is the reactive power at branch $i, U[i]$ is the voltage at branch $i$ after DG injection, $R[i]$ is the resistance of branch $i$, and $m$ is the number of branches in the distribution network.

In the previous three optimization models, the fuel cost and the pollutant emission penalty function $F_{1}(x)$ and the system loss function $F_{3}(x)$ should be minimized, whereas the cost-saving function $F_{2}(x)$ should be maximized.

2.2. Constraints. Three constraint conditions are considered in the optimization model, which includes constraints of power flow equations, nodal voltage, and DG capacity.
2.2.1. Equality Constraints. The constraint of power flow equations is described as follows:

$$
\begin{gathered}
P_{\mathrm{DG}_{i}}-P_{d_{i}}=V_{i} \sum_{j=1}^{N} V_{j}\left(G_{i j} \cos \theta_{i j}+B_{i j} \sin \theta_{i j}\right), \\
Q_{\mathrm{DG}_{i}}-Q_{d_{i}}=V_{i} \sum_{j=1}^{N} V_{j}\left(G_{i j} \sin \theta_{i j}-B_{i j} \cos \theta_{i j}\right),
\end{gathered}
$$

where $P_{\mathrm{DG}_{i}}$ and $Q_{\mathrm{DG}_{i}}$ are active and reactive generation outputs, whereas $P_{d_{i}}$ and $Q_{d_{i}}$ are the active and reactive load at bus $i$, respectively, $G_{i j}$ and $B_{i j}$ are the transfer conductance and susceptance between bus $i$ and $j$, respectively, and $N$ is the number of buses.

\subsubsection{Inequality Constraints}

Generation limits:

$$
\begin{gathered}
P_{\mathrm{DG}_{i}}^{\min } \leq P_{\mathrm{DG}_{i}} \leq P_{\mathrm{DG}_{i}}^{\max }, \\
Q_{\mathrm{DG}_{i}}^{\min } \leq Q_{\mathrm{DG}_{i}} \leq Q_{\mathrm{DG}_{i}}^{\max } .
\end{gathered}
$$

Load bus voltage constraints:

$$
V_{i \min } \leq V_{i} \leq V_{i \max }
$$

Thermal limits:

$$
\begin{aligned}
\left|S_{i j}\right| & =\left|V_{i}^{2} G_{i j}-V_{i} V_{j}\left(G_{i j} \cos \theta_{i j}+B_{i j} \sin \theta_{i j}\right)\right| \\
& \leq S_{i j}^{\max } .
\end{aligned}
$$

In the inequality constraints, $P_{\mathrm{DG}_{i}}^{\min }, P_{\mathrm{DG}_{i}}^{\max }, Q_{\mathrm{DG}_{i}}^{\min }$, and $Q_{\mathrm{DG}_{i}}^{\max }$ are the lower/upper active and reactive generating unit limits of DG, respectively. $S_{i j}^{\max }$ is the apparent power thermal limit of the circuit between buses $i$ and $j$.

There is always a limit on penetration of DG for a distribution power system to ensure reliability. Different countries have different penetration factor values. The penetration factor indicates the aggregated DG rating on an electric power system (EPS) feeder, divided by the peak EPS feeder load. If we assume that the maximum DG penetration factor is $25 \%$, then the maximum injected DG capacity should be limited to $25 \%$ of the maximum total load in the distribution network, which can be described as follows:

$$
\sum_{i=1}^{n} P_{\mathrm{DG}_{i}} \leq 0.25 S^{\max }, \quad\left(i \in \Phi_{S}\right),
$$

where $P_{\mathrm{DG}_{i}}$ is the DG access capacity at node $i$ and $S^{\max }$ is the maximum load capacity of distribution network.

2.3. Overview Formulation. Aggregating the objectives and constraints, the problem can be formulated as a nonlinear programming problem as follows:

$$
\begin{array}{ll}
\operatorname{minimize} & {\left[F_{1}(x), F_{2}(x), \ldots, F_{k}(x)\right],} \\
\text { subject to } & h_{i}(x)=0, \quad i=1, \ldots, p, \\
& g_{i}(x) \leq 0, \quad i=1, \ldots, n,
\end{array}
$$


where $k$ is the number of objectives and $x$ is the vector of dependent variables consisting of slack bus power output and DG active power out $P_{\mathrm{DG}_{i}}$, load bus voltage $V_{i}$, and generator reactive power outputs $Q_{D_{G}}$. Thus, $x$ can be expresses as follows:

$$
x=\left[P_{\mathrm{DG}_{1}}, \ldots, P_{\mathrm{DG}_{N G}}, V_{L_{1}}, \ldots, V_{L_{N L}}, Q_{D G 1}, \ldots, Q_{\mathrm{DG}_{N G}}\right]^{T}
$$

where $n$ is the number of inequality constraints, $p$ is number of equation constraints, $k$ is the number of objectives, and $N G$ is the number of DG units.

\section{A Pareto-Based Algorithm and Additional Concepts}

3.1. Concepts of Dominated, Nondominated, and Pareto Set. Multi-objective optimization can be expressed as

$$
\min f_{i}(x), \quad i=1,2, \ldots, m, x \in \chi,
$$

where $f_{i}(x)$ denotes the $i$ th objective function, $m$ is the number of objectives, and $\chi$ represents the feasible search space.

Definition 1. A solution $x_{1}$ is said to dominate $x_{2}$ (denoted by $\left.x_{1} \prec x_{2}\right)$ if and only if

$$
\begin{aligned}
\forall i & \in\{1,2, \ldots, m\}: f_{i}\left(x_{1}\right) \leq f_{i}\left(x_{2}\right) \\
& \wedge \exists j \in\{1,2, \ldots, m\}: f_{j}\left(x_{1}\right)<f_{j}\left(x_{2}\right) .
\end{aligned}
$$

Definition 2. For $S=\left\{x_{i}, i=1, \ldots, n\right\}$, solution $x$ is said to be a nondominated solution (Pareto solution) of set $S$ if $x \in S$, and there is no solution $x^{\prime} \in S$ for which $x^{\prime}$ dominates $x$.

Definition 3. Assume that set $P$ contains all the nondominated solutions of $S$, then $\mathrm{PF}=\left\{v \mid v=\left[f_{1}(x), f_{2}(x)\right.\right.$, $\left.\left.\ldots, f_{m}(x)\right]^{T}, x \in P\right\}$ is a Pareto front of set $S$.

3.2. Basic Pareto-Based Evolutionary Algorithm. The traditional Pareto-based evolutionary algorithm is shown in Figure 1. The detailed algorithm procedure is explained in [16]. The main improvements on the Pareto-based algorithm can be generalized as follows. The penalty function is established to constrain the solution of the objective function. The adaptive crossover and mutation are adopted in the evolution process, which improves the probability of global optimization. The simulated annealing algorithm is added to the iterative process, so that the algorithm is able to seek the optimal solution globally and rapidly converges to the optimal solution.

\section{Proposed Improved Pareto Evolutionary Algorithm}

4.1. Overview. To solve the difficulties in traditional optimization techniques, a new evolutionary population-based searching technique is proposed to solve the multiobjective optimization problem based on SPEA2 [17, 18].

4.2. Initialization. In the improved SPEA2, an individual $i$ at generation $G$ is a multidimensional vector $x_{i}^{G}=$ $\left(x_{i, 1}, \ldots, x_{i, D}\right)$. The population is initialized by randomly generating individuals as

$$
\begin{array}{r}
x_{i, k}^{G}=x_{k_{\min }}+\operatorname{rand}[0,1] \times\left(x_{k_{\max }}-x_{k_{\min }}\right), \\
i \in\left[1, N_{p}\right], k \in[1, D],
\end{array}
$$

where $N_{p}$ is the population size and $D$ is the number of control variables. Each variable $k$ in a solution vector $i$ in the generation $G$ initialized within its boundaries $x_{k_{\min }}$ and $x_{k_{\max }}$.

4.3. Fitness Evaluation. The objective of each solution $F_{1}(x), F_{2}(x), \ldots, F_{k}(x)$ will be computed. The individual fitness values in both the population-based set Pop and nondominated archive set NDSet will be calculated based on (17). The mismatch of each constraint value is multiplied by a large value and added to all objectives to remove infeasible solutions. The methodology is to evaluate the feasible solutions according to the value of objective function and remove the infeasible solutions according to the constraints.

The individual's fitness $M(i)$ will be obtained from the sum of the primary fitness value $R(i)$ and the density $D(i)$ as follows:

$$
M(i)=R(i)+D(i)
$$

where $R(i)=\sum_{j \in P o p+N D S e t, j>i} S(j), S(j)$ is the objective evaluation for the individual $j$. ( $>$ indicates a dominated relation, $x_{i}>x_{j}$ indicates $x_{i}$ dominates $x_{j}, x_{i}$ is nondominated, and $x_{j}$ is dominated).

$D(i)=1 /\left(\sigma_{i}^{k}+2\right), k=\sqrt{N+\bar{N}} \cdot \sigma_{i}^{k}$ represents the objective space distance between individual $i$ in Pop and the $k$ th nearest neighbor individual in the NDSet. It is the Knearest neighbor (KNN) method, and the distance between the individual $i$ in Pop and the other individuals in the NDSet need to be computed, and then, the distance value can be sorted.

4.4. Adaptive Crossover and Mutation Probability. The selection of crossover probability $P_{c}$ and mutation probability $P_{m}$ dominates the solution process. $P_{c}$ and $P_{m}$ determine the generation speed and the probability of new individuals, respectively. If $P_{c}$ exceeds the threshold, the generation speed of the new population will be quicker, which means that there is a stronger capability to explore new space. If $P_{c}$ is extremely small, the search process will be quite slow. If $P_{m}$ is too large, the search process will be more random. The adaptive value of 


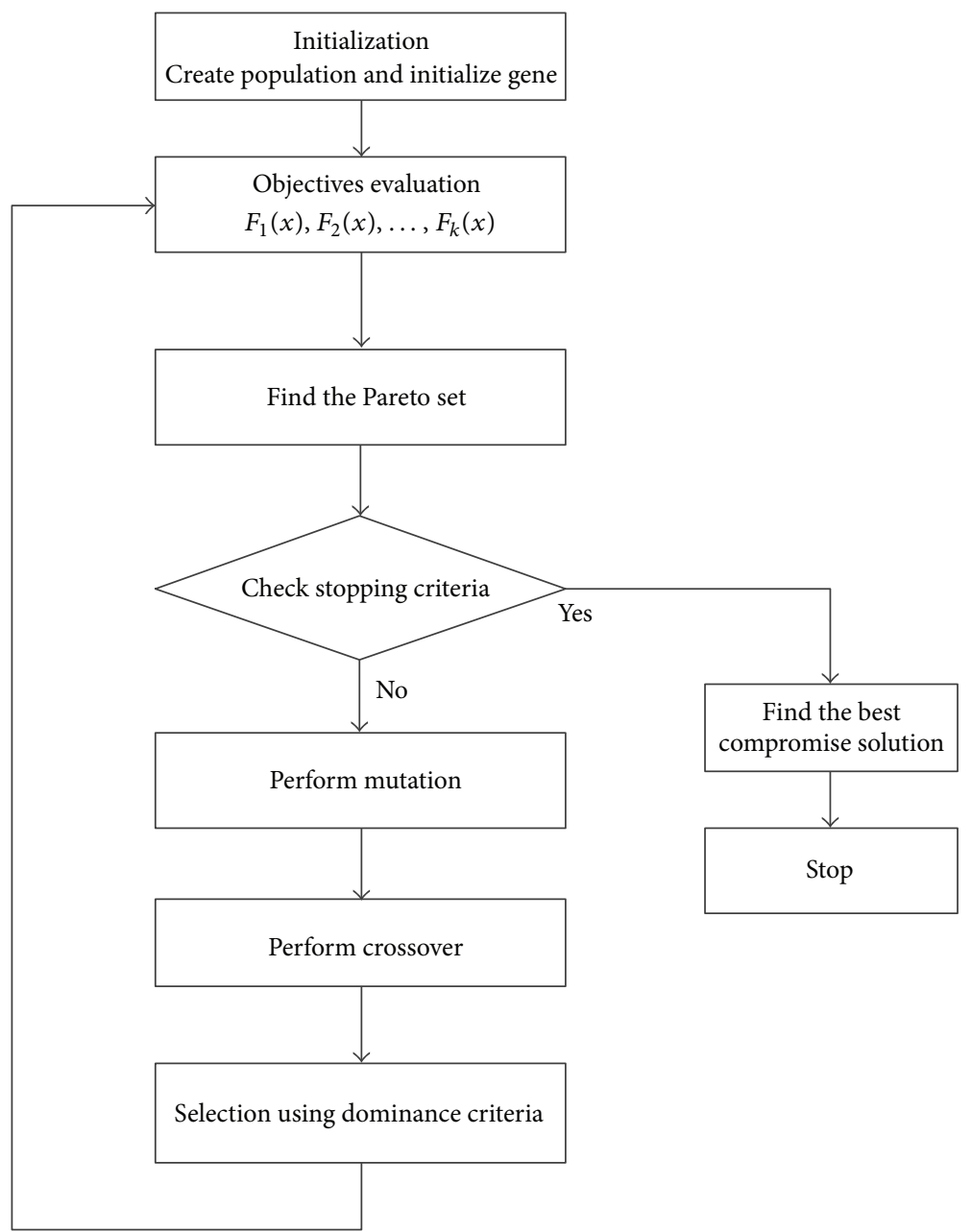

FIgURE 1: Flowchart of traditional Pareto-based approach.

$P_{c}$ and $P_{m}$ is obtained from the following evaluated equations:

$$
\begin{gathered}
P_{c}= \begin{cases}\frac{P_{c_{1}}\left(M_{\mathrm{avg}}-M^{\prime}\right)+P_{\mathcal{c}_{2}}\left(M^{\prime}-M_{\mathrm{min}}\right)}{\mathrm{M}_{\mathrm{avg}}-\mathrm{M}_{\mathrm{min}}}, & M^{\prime}<M_{\mathrm{avg}}, \\
\frac{P_{\mathcal{c}_{2}}\left(M_{\max }-M^{\prime}\right)+P_{\mathcal{c}_{3}}\left(M^{\prime}-M_{\mathrm{avg}}\right)}{M_{\max }-M_{\mathrm{avg}}}, & M^{\prime} \geq M_{\mathrm{avg}},\end{cases} \\
P_{m}= \begin{cases}\frac{P_{m_{1}}\left(M_{\mathrm{avg}}-M\right)+P_{m_{2}}\left(M-M_{\min }\right)}{M_{\mathrm{avg}}-M_{\min }}, & M<M_{\mathrm{avg}}, \\
\frac{P_{m_{2}}\left(M_{\max }-M\right)+P_{m_{3}}\left(M-M_{\mathrm{avg}}\right)}{M_{\max }-M_{\mathrm{avg}}}, & M \geq M_{\mathrm{avg}},\end{cases}
\end{gathered}
$$

where $M_{\mathrm{avg}}$ is the average fitness value, $M_{\max }$ is the biggest fitness value, $M^{\prime}$ is the bigger fitness value of two genes in the crossover process, $M$ is the mutating individual's fitness value, and the constants $P_{c_{1}}, P_{c_{2}}, P_{c_{3}}, P_{m_{1}}, P_{m_{2}}, P_{m_{3}} \in[0,1]$, $P_{c_{1}}>P_{c_{2}}>P_{c_{3}}, P_{m_{1}}>P_{m_{2}}>P_{m_{3}}$.
4.5. Pareto Optimal Selection Using Fuzzy Set Theory. In this paper, fuzzy set theory is used to select the optimal solution set among the obtained multiobjective solution sets. Fuzzy sets are sets whose elements have degrees of membership. Fuzzy set theory permits the gradual assessment of the membership of elements in a set. This membership is described with the aid of a membership function valued in the real unit interval $[0,1]$.

First, define a linear membership function $\tau_{i}$ as the weight of target $i$ in a solution:

$$
\tau_{\mathrm{i}}= \begin{cases}1 & F_{i}=F_{i}^{\min }, \\ \frac{F_{i}^{\max }-F_{i}}{F_{i}^{\max }-F_{i}^{\min }} & F_{i}^{\min }<F_{i}<F_{i}^{\max }, \\ 0 & F_{i} \leq F_{i}^{\max },\end{cases}
$$

where $F_{i}^{\max }$ is the maximum of $i$ th objective function, $F_{i}^{\min }$ is the minimum of $i$ th objective function, and $F_{i}$ is the solution of $i$ th objective. The previous equation provides a measure of the degree of satisfaction for each objective function for a particular solution. 
The dominant function $\tau_{k}$ for each nondominated solution $k$ in Pareto solution set is calculated as follows:

$$
\tau_{k}=\frac{\sum_{i=1}^{N_{0}} \tau_{k}^{i}}{\sum_{j=1}^{u} \sum_{i=1}^{N_{0}} \tau_{j}^{i}},
$$

where $u$ is the number of the Pareto solution set and $N_{0}$ is the number of the optimization objectives.

Because the value of $\tau_{k}$ determines the capability of the solution, the solution with maximum $\tau_{k}$ will be Pareto optimal. Moreover, the feasible priority sequence can be obtained by the value of $\tau_{k}$, in descending order.

The best Pareto optimal solution is the one achieving the maximum membership function $\tau_{k}$, as shown in (20).

4.6. Simulated Annealing in Population-Based Individual Selection. Simulated annealing (SA) is a generic probabilistic metaheuristic for the global optimization problem of locating a good approximation to the global optimum of a given function in a large search space. It is often used when the search space is discrete. Here, SA is utilized in the individual selection.

Based on the individuals after selection, crossover and mutation steps, the simulated annealing operation is performed on the individuals of the population. The two genes in each individual will be selected and disturbed randomly. Then, the new individual will be evaluated to form new fitness values. If the fitness value of a new individual is larger than the old value, then the old individual will be replaced by the new individual. If the fitness value of the new individual is smaller than the old value, the new individual can also be accepted using the following probability:

$$
p\left(T_{k+1}\right)= \begin{cases}1 & \left(M_{k+1}>M_{k}\right), \\ \exp \left(-\frac{M_{k+1}-M_{k}}{T_{k+1}}\right) & \left(M_{k+1} \leq M_{k}\right), \\ T_{k+1}=\alpha T_{k},\end{cases}
$$

where $M_{k+1}$ and $M_{k}$ are the fitness of the new individual and old individual, respectively, $p\left(T_{k+1}\right)$ is the acceptance probability at $T_{k+1}$ temperature, and $\alpha$ is the temperature descending coefficient.

4.7. Convergence Condition. The iterative procedure can be terminated when any of the following conditions are met: (1) the true Pareto front is obtained, and (2) the iteration number of the algorithm reaches the predefined maximum number of iterations. However, the true Pareto front will not be known in advance in most practical multiobjective problems, so the convergence condition is to iterate to a predefined maximal iteration number.

4.8. Flowchart of Proposed Algorithm. The flow chart of the proposed algorithm is illustrated in Figure 2. As shown in Figure 2, the steps of the proposed evolutionary algorithm are described as follows.
Step 1. Generate an initial set Pop randomly and an empty archive set NDSet over the problem space; initialize the parameters of the population size $N$, nondominated archive size $\bar{N}$, and maximum generation's number $T$.

Step 2. Establish the penalty function to constrain each objective function, and then form new objective functions.

Step 3. Compute the fitness of individual in both the population-based set Pop and the nondominated archive set NDSet. The objective of each solution $F_{1}(x), F_{2}(x), \ldots, F_{k}(x)$ will be computed.

Step 4. Duplicate the nondominated individuals in both the population and nondominated archive set to a new archive set NDSet_new, if the size of NDSet_new exceeds $\bar{N}$, then reduce NDSet_new by means of the truncation operator; otherwise, fill NDSet_new with dominated individuals in Pop and NDSet.

Step 5. Evaluate if the nondominated set NDSet new exceeds the predefined size $\bar{N}$. If the size of NDSet new is larger than $\bar{N}$, then truncate the nondominated individuals; otherwise, continue to Step 6.

Step 6. Copy the superior dominated individual to NDSet_new.

Step 7. Evaluate the convergence criteria. If the iteration number $t \geq T$, terminate the iteration to obtain the Pareto optimal solution and output the best solution; otherwise, set $t=t+1$, and continue to Step 8 .

Step 8. Perform adaptive crossover and mutation operation on the individuals of Pop.

Step 9. Perform a simulated annealing operation, and then go to Step 3.

\section{Experiments and Results}

To demonstrate the effectiveness of the proposed method, the algorithm in Section 4 was implemented to obtain solutions for optimal active power dispatch of DG. The IEEE 33 bus distribution system was examined, and three objectives were considered in this study. These objectives were fuel cost/pollutant emission, transmission line loss, and cost savings on bills using DG. Photovoltaic (PV) panels, diesel turbine, and wind turbine distribution are injected into bus 7 , bus 17, bus 21, and bus 32, respectively, as shown in Figure 3. In this paper, $P_{c}$ and $P_{m}$ are defined as follows: $P_{c_{1}}=0.4$, $P_{c_{2}}=0.3$, and $P_{c_{3}}=0.2$ and $P_{m_{1}}=0.2, P_{m_{2}}=0.1$, and $P_{m_{3}}=0.05$. The maximum iteration number is set to 200 . The proposed algorithm was coded in $\mathrm{C}++$ and run on an Intel i5-3210M 2.5 GHz notebook with 4 GB RAM.

5.1. Energy Utilization Cost. Among the four DG units, only the two diesel turbine DG units have fuel cost. Because it would be difficult for market players to accept/implement 


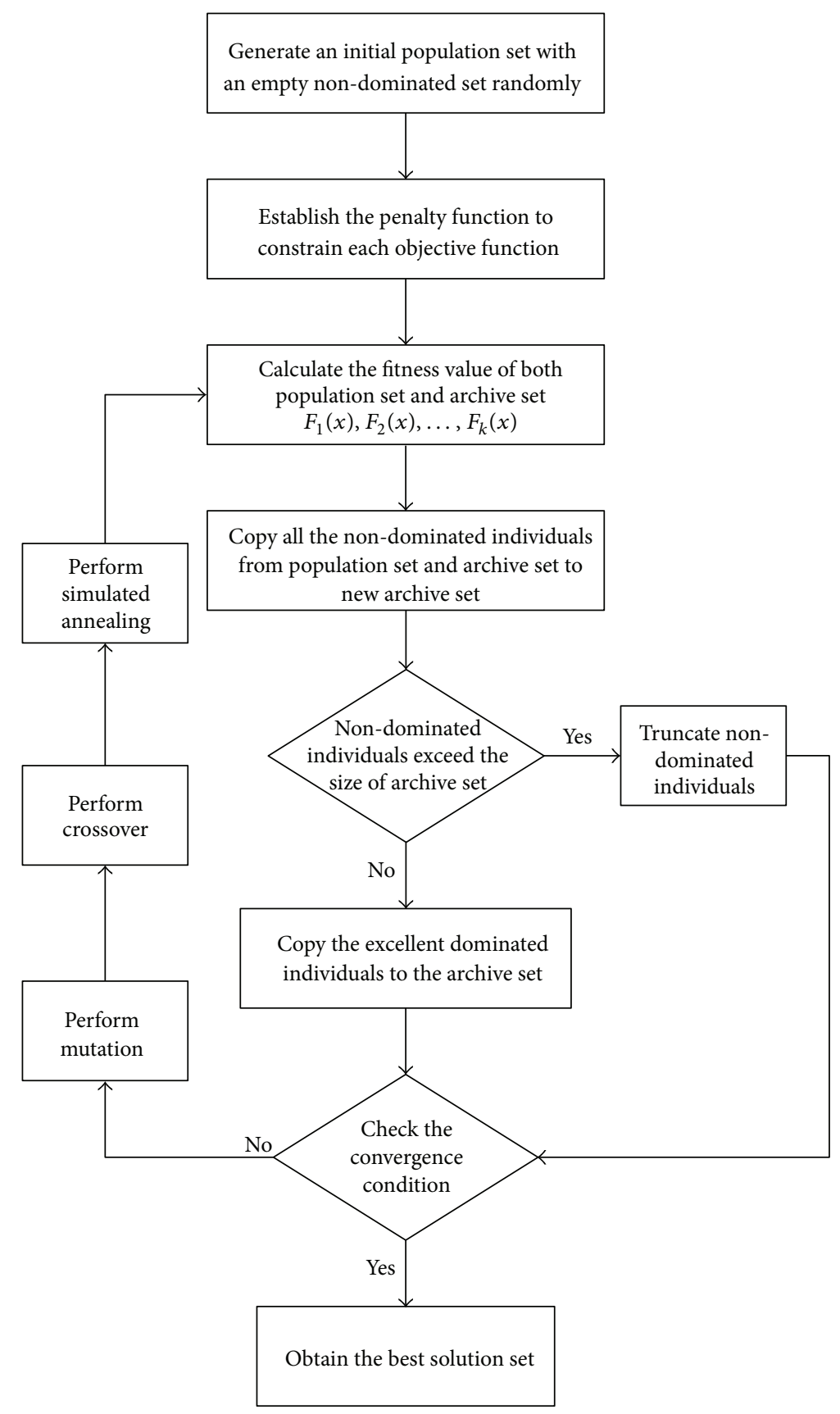

FIGURE 2: Flowchart of improved Pareto-based evolutionary algorithm.

a central cost-based dispatch in the distribution system including DG units, the cost of fossil-fuel consumed by micro diesel turbine is calculated as follows:

$$
C_{R}=\sum_{i=1}^{n} f\left(P_{i}^{t}\right) C_{i}
$$

where $C_{i}$ is fuel price at power unit $i$ and $f\left(P_{i}^{t}\right)$ is the required fuel quantity for power unit $i$ at the moment $t$.
5.2. Penalty on Pollutant Emission. As global environmental pollution is growing, optimizing power generation and pollutant emission costs are two conflicting goals. These goals present a restrictive and coordinated relationship. Environmental cost mainly refers to the fines related to pollutant emission. Tables 1 and 2 show the pollutant emission data for various DG units and the standard electric power industry pollution fines, respectively. In reference report [19], there are similar pollutant cost coefficients for distributed generation. Based on the DG unit output 


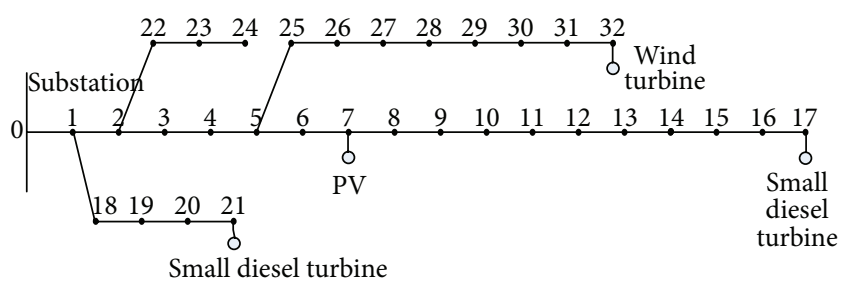

FIGURE 3: Schematic diagram of the IEEE 33-bus system with 4 DG units.

TABLE 1: The pollutant emission rate of DG units ( $\mathrm{g} / \mathrm{kWh}$ ).

\begin{tabular}{lcccc}
\hline $\begin{array}{l}\text { Pollutant } \\
\text { emission }\end{array}$ & $\begin{array}{c}\text { Coal } \\
\text { generation }\end{array}$ & Diesel engine & PV panel & $\begin{array}{c}\text { Wind } \\
\text { turbine }\end{array}$ \\
\hline $\mathrm{NO}_{x}$ & 6.46 & 4.3314 & 0 & 0 \\
$\mathrm{CO}_{2}$ & 1070 & 232.0373 & 0 & 0 \\
$\mathrm{CO}$ & 1.55 & 2.3204 & 0 & 0 \\
$\mathrm{SO}_{2}$ & 9.93 & 0.4641 & 0 & 0 \\
\hline
\end{tabular}

TABLE 2: Standard pollutant emission penalties $(\$ / \mathrm{kg})$.

\begin{tabular}{cccc}
\hline $\mathrm{SO}_{2}$ & $\mathrm{NO}_{x}$ & $\mathrm{CO}_{2}$ & $\mathrm{CO}$ \\
\hline 0.75 & 1.00 & 0.002875 & 0.125 \\
\hline
\end{tabular}

following multiple-objective optimization, the quantity of pollutant emission can be obtained.

5.3. Optimization Results. Using the optimization model developed in Section 3, the optimized output of four DG units over $24 \mathrm{~h}$ and the power system losses after DG unit injection are shown in Figures 4 and 5, respectively. As shown in Figure 4, the four DG units have different active power outputs at different time periods in a day. The diesel power output will increase when the solar and wind power outputs are at a low level. When the PV output and wind power output increase to the peak, it will stop increasing and stay at the peak power output, and then, the diesel power output will gradually decrease.

As shown in Figure 5, the line losses greatly decrease DG unit penetration into the distribution system. From the hours 8 to 17 , the total output of the four DG units provides enough active power, which improves the voltage quality and reduces the line loss.

The forecasted and optimized solar power outputs based on the computed results are shown in Figure 6, and the forecasting and optimized wind power output are shown in Figure 7. The forecasted PV and wind generation values are based on historical distribution system data. Because of the cooperative optimization, the optimal real power values of PV and wind generation are smaller than the forecasted values in the peak time period.

Assuming that the coal consumption from the power plant is $0.35 \mathrm{~kg} / \mathrm{kWh}$ and the highest coal price is 0.124 $\$ / \mathrm{kg}$, the cost savings for coal consumption by using clean energy is illustrated in Figure 8, which shows that these

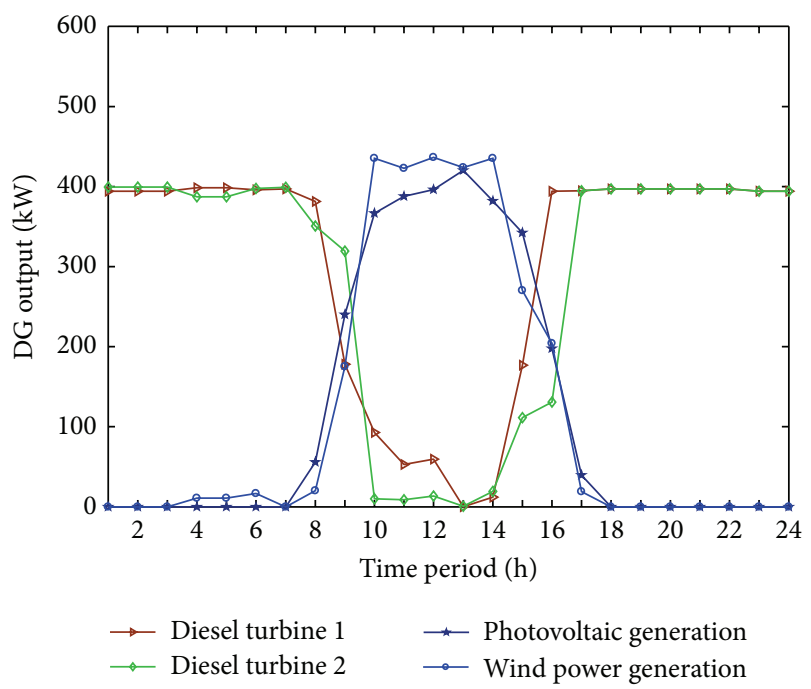

FIgURE 4: The optimized output of four DG units in 24 hours.

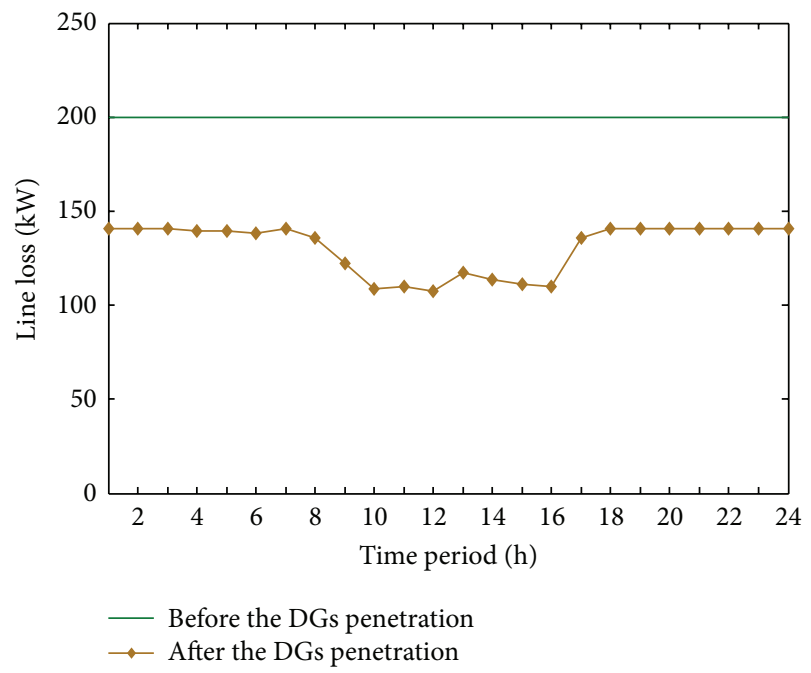

FIGURE 5: The power system loss before and after DG unit injection.

increases in solar and wind power output results in greater coal consumption cost savings.

A pollutant emission penalty reduction curve was obtained based on data from Tables 1 and 2, and the hourly penalty reduction for pollutant emission is shown in Figure 9. As there is no pollutant emission for solar and wind power generation, when the output of new energy power supply increases, the environment cost will decrease significantly.

Assuming that the time-of-use price is $0.095 \$ / \mathrm{kWh}$ for peak time from $6: 00 \mathrm{am}$ to $22: 00 \mathrm{pm}$ and $0.054 \$ / \mathrm{kWh}$ in other period, the cost saving for the electrical bills of users per hour is shown in Figure 10. Because the price is at a high level from 6:00 am to 18:00 pm, the bill saving increases with the increase of PV and wind power output. The results from the case study demonstrates that the system loss is greatly reduced by $65 \%$, so that the users, in total, can save $\$ 1,671$ per day on their electricity bills, and power plants can save 


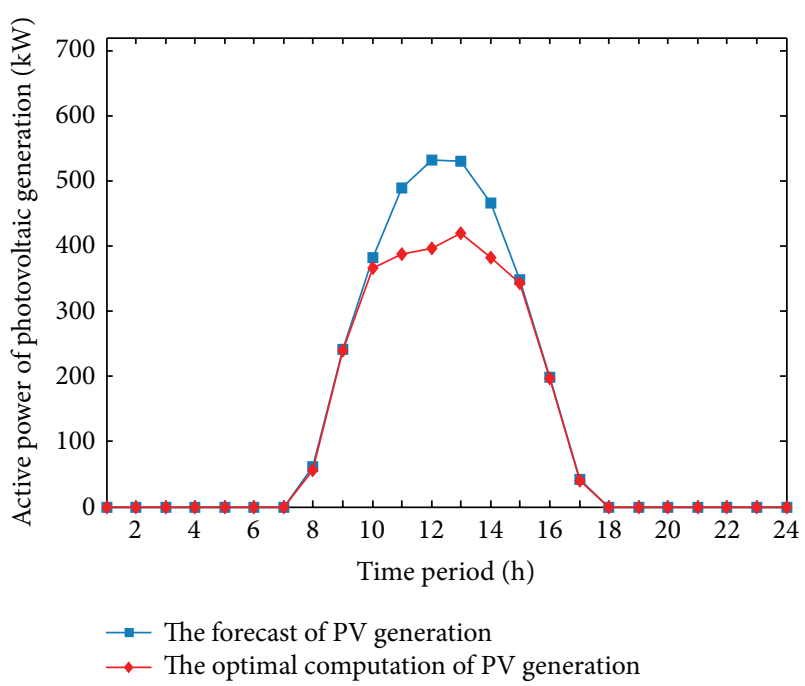

FIGURE 6: The comparison of forecasted PV value and the computed optimal PV value.

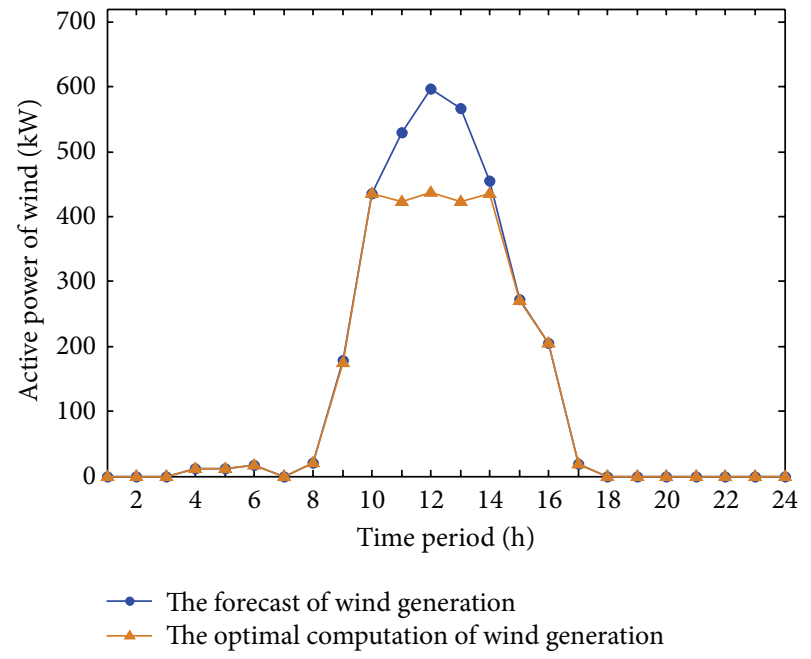

FIgURE 7: The comparison of forecasted wind values and the computed optimal value.

$\$ 870$ and $\$ 9,906$ on their coal costs and pollutant emission penalties per day, respectively.

5.4. Comparison of Different Algorithms. The proposed algorithm was compared with the SPEA2 [17] and the particle swarm optimization method [20] and NSGA-II [21, 22]. The IEEE 33-bus system with four DG units was utilized as an example for this comparison. The load data at hour 11 is selected as the basic load data. The convergence condition was that the iteration number exceeded the preset maximum iteration number, which was set to 200. In PSO, the cognitive ratio and social ratio are all equal to 2.0. The number of swarm particles is 100. In NSGA-II, the crossover ratio is set to 0.8 , and the mutation ratio is set to 0.2 . The size of population is set to 100 .

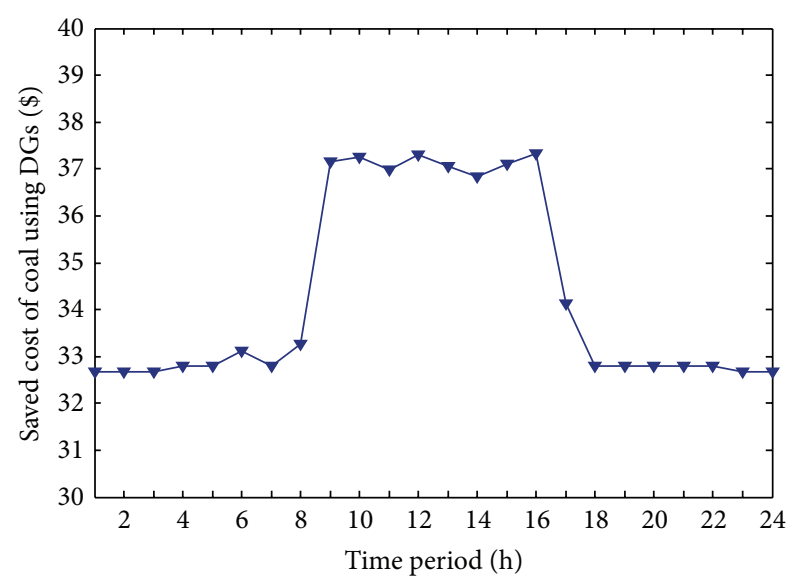

FIGURE 8: Hourly cost savings on coal consumption.

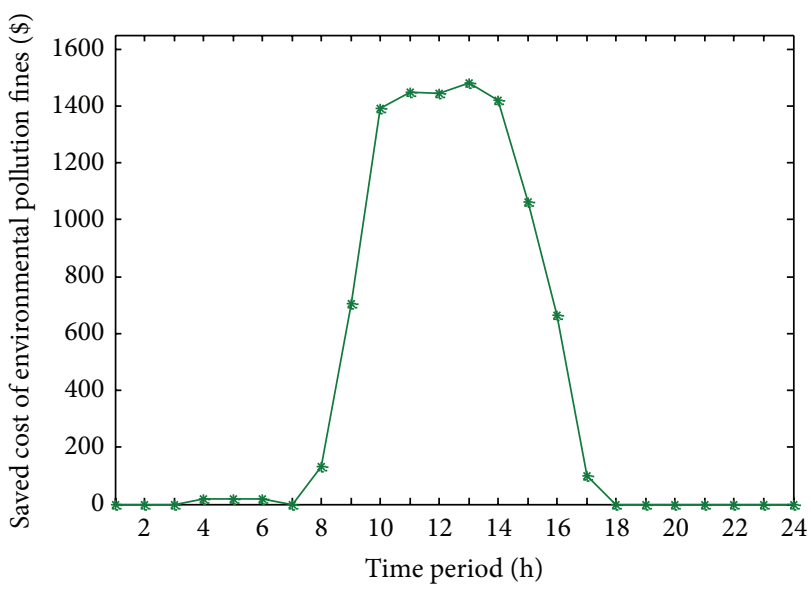

FiguRE 9: Hourly penalty reduction for pollutant emission.

Table 3 shows that the proposed algorithm performs better than the SPEA2 and the PSO algorithms with respect to calculating the multiple objective objectives in the same limited iterations, and the proposed algorithm has better convergence speed than SPEA2 and PSO because simulated annealing is added in addition to the adaptive crossover and mutation operations. Compared with NSGA-II, the proposed algorithm has approximate speed in searching the Pareto front.

\section{Conclusion}

This paper presented an improved Pareto-based evolutionary algorithm, which increases the global optimization ability with a simulated annealing iterative process and fuzzy set theory, to solve the multiobjective optimization problem for a distribution power system. The proposed algorithm was utilized to optimize a model of DG unit injection with objectives of maximizing the utilization of DG while minimizing the system loss and environmental pollution. The results indicate that the proposed optimization is applicable to practical multiobjective optimization problems that take 


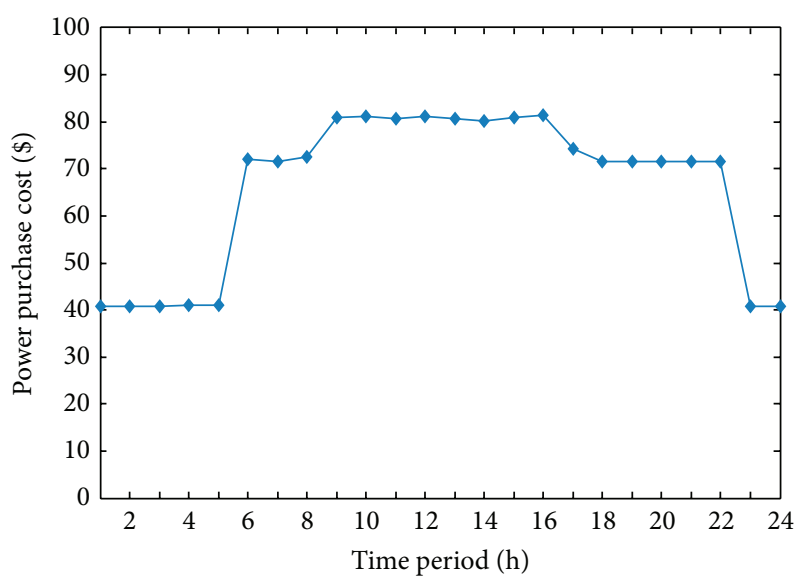

FIGURE 10: Hourly bill saving for consumers with DG unit injection.

TABLE 3: Comparison of different algorithms.

\begin{tabular}{lccccc}
\hline & Iterations & Time & $\begin{array}{c}\text { Min } \\
F_{1}(x)\end{array}$ & $\begin{array}{c}\text { Max } \\
F_{2}(x)\end{array}$ & $\begin{array}{c}\text { Min } \\
F_{3}(x)\end{array}$ \\
\hline $\begin{array}{l}\text { Proposed } \\
\text { algorithm }\end{array}$ & 200 & $34 \mathrm{~s}$ & $\$ 1535.3$ & $\$ 86.0$ & $114.5 \mathrm{~kW}$ \\
SPEA2 & 200 & $42 \mathrm{~s}$ & $\$ 1568.5$ & $\$ 84.5$ & $129.0 \mathrm{~kW}$ \\
PSO & 200 & $39 \mathrm{~s}$ & $\$ 1589.6$ & $\$ 82.6$ & $131.2 \mathrm{~kW}$ \\
NSGA-II & 200 & $36 \mathrm{~s}$ & $\$ 1573.9$ & $\$ 83.2$ & $125.2 \mathrm{~kW}$ \\
\hline
\end{tabular}

into considering the requirements from utilities, consumers, and the environment.

With respect to the state of the art, the improvements from this new multiobjective optimization method can be listed as follows: (1) the ability to search an entire set of Pareto optimal solutions is enhanced by using SA, which is proven by the comparison experiments, and (2) the Pareto front converges to better optimum set of solutions using the proposed algorithm. Future work will be focused on probabilistic evaluation and optimization that considers multiple DG units and load profile in distribution systems.

\section{References}

[1] E. Zitzler and L. Thiele, "Multiobjective evolutionary algorithms: a comparative case study and the strength Pareto approach," IEEE Transactions on Evolutionary Computation, vol. 3, no. 4, pp. 257-271, 1999.

[2] M. A. Abido and N. A. Al-Ali, "Multi-objective optimal power flow using differential evolution," Arabian Journal for Science and Engineering, vol. 37, no. 4, pp. 991-1005, 2012.

[3] M. Varadarajan and K. S. Swarup, "Solving multi-objective optimal power flow using differential evolution," IET Generation, Transmission and Distribution, vol. 2, no. 5, pp. 720-730, 2008.

[4] C. A. Coello Coello, "Evolutionary multi-objective optimization: a historical view of the field," IEEE Computational Intelligence Magazine, vol. 1, no. 1, pp. 28-36, 2006.

[5] M. A. Abido, "Multiobjective evolutionary algorithms for electric power dispatch problem," IEEE Transactions on Evolutionary Computation, vol. 10, no. 3, pp. 315-329, 2006.
[6] G. Celli, E. Ghiani, S. Mocci, and F. Pilo, "A multiobjective evolutionary algorithm for the sizing and siting of distributed generation," IEEE Transactions on Power Systems, vol. 20, no. 2, pp. 750-757, 2005.

[7] T. Niknam, M. R. Narimani, J. Aghaei et al., "Improved particle swarm optimisation for multi-objective optimal power flow considering the cost, loss, emission and voltage stability index," IET Generation, Transmission and Distribution, vol. 6, no. 6, pp. 515-527, 2012.

[8] P. S. Manoharan, P. S. Kannan, S. Baskar, and M. W. Iruthayarajan, "Penalty parameter-less constraint handling scheme based evolutionary algorithm solutions to economic dispatch," IET Generation, Transmission and Distribution, vol. 2, no. 4, pp. 478490, 2008.

[9] Y. Wang and Z. Cai, "Combining multiobjective optimization with differential evolution to solve constrained optimization problems," IEEE Transactions on Evolutionary Computation, vol. 16, no. 1, pp. 117-134, 2012.

[10] A. Darvishi, A. Alimardani, and S. H. Hosseinian, "Fuzzy multi-objective technique integrated with differential evolution method to optimise power factor and total harmonic distortion," IET Generation, Transmission and Distribution, vol. 5, no. 9, pp. 921-929, 2011.

[11] A. Bhattacharya and P. K. Chattopadhyay, "Hybrid differential evolution with biogeography-based optimization for solution of economic load dispatch," IEEE Transactions on Power Systems, vol. 25, no. 4, pp. 1955-1964, 2010.

[12] T. Niknam and H. Doagou-Mojarrad, "Multiobjective economic/emission dispatch by multiobjective $\theta$-particle swarm optimisation," IET Generation, Transmission and Distribution, vol. 6, no. 5, pp. 363-377, 2012.

[13] A. I. Aly, Y. G. Hegazy, and M. A. Alsharkawy, "A simulated annealing algorithm for multi-objective distributed generation planning," in IEEE Power and Energy Society General Meeting (PES '10), pp. 1-7, July 2010.

[14] R. S. Maciel and A. Padilha-Feltrin, "Distributed generation impact evaluation using a multi-objective tabu search," in Proceedings of the 15th International Conference on Intelligent System Applications to Power Systems (ISAP '09), pp. 1-5, November 2009.

[15] E. B. Cano, "Utilizing fuzzy optimization for distributed generation allocation," in Proceedings of the 10 Annual International Conference (TENCON '07), pp. 1-4, Taipei, China, October 2007.

[16] C. A. Coello Coello, G. B. Lamont, and D. A. Van Veldhuizen, Evolutionary Algorithms for Solving Multi-Objective Problems, Genetic and Evolutionary Computation Series, Springer, New York, NY, USA, 2nd edition, 2007.

[17] Z. Eckart, M. Laumanns, and L. Thiele, "SPEA2: improving the Strength Pareto evolutionary algorithm," TIK Report 103, Swiss Federal Institute of Technology (ETH), Zurich, Switzerland, 2001.

[18] W. Sheng, Y. Liu, X. Meng, and T. Zhang, "An Improved Strength Pareto Evolutionary Algorithm 2 with application to the optimization of distributed generations," Computers and Mathematics with Applications, vol. 64, no. 5, pp. 944-955, 2012.

[19] Y. H. Wan and S. Adelman, Distributed Utility Technology Cost, Performance, and Environmental Characteristic, United States, National Renewable Energy Laboratory, 1995.

[20] M. AlHajri, Sizing and placement of distributed generation in electrical distribution systems using conventional and heuristic optimization methods [Ph.D. Dissertation], Dalhousie University, Halifax, Canada, 2009. 
[21] K. Deb, A. Pratap, S. Agarwal, and T. Meyarivan, "A fast and elitist multiobjective genetic algorithm: nSGA-II," IEEE Transactions on Evolutionary Computation, vol. 6, no. 2, pp. 182197, 2002.

[22] http://www.tik.ee.ethz.ch/sop/pisa/selectors/nsga2/?page= nsga2.php. 


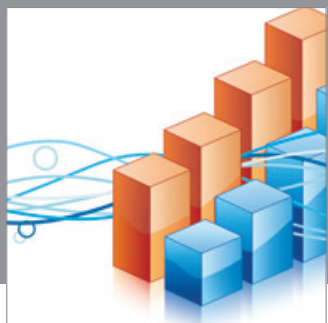

Advances in

Operations Research

mansans

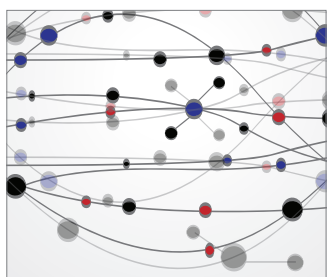

The Scientific World Journal
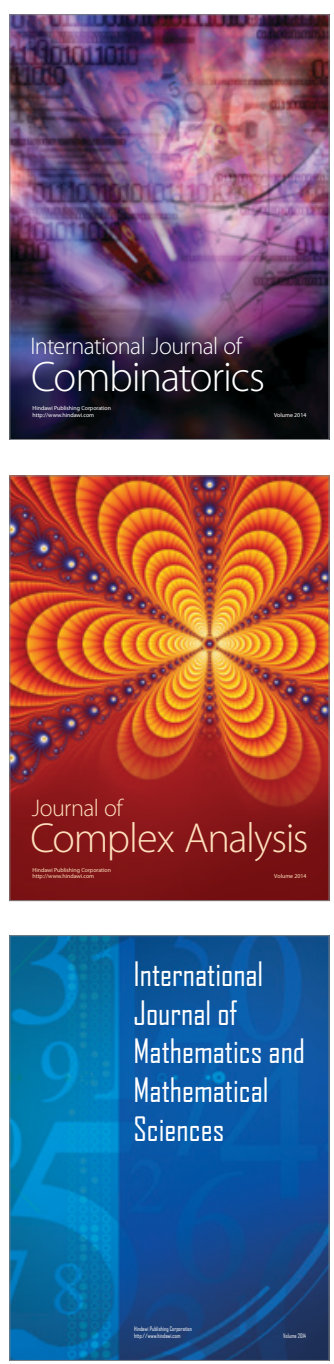
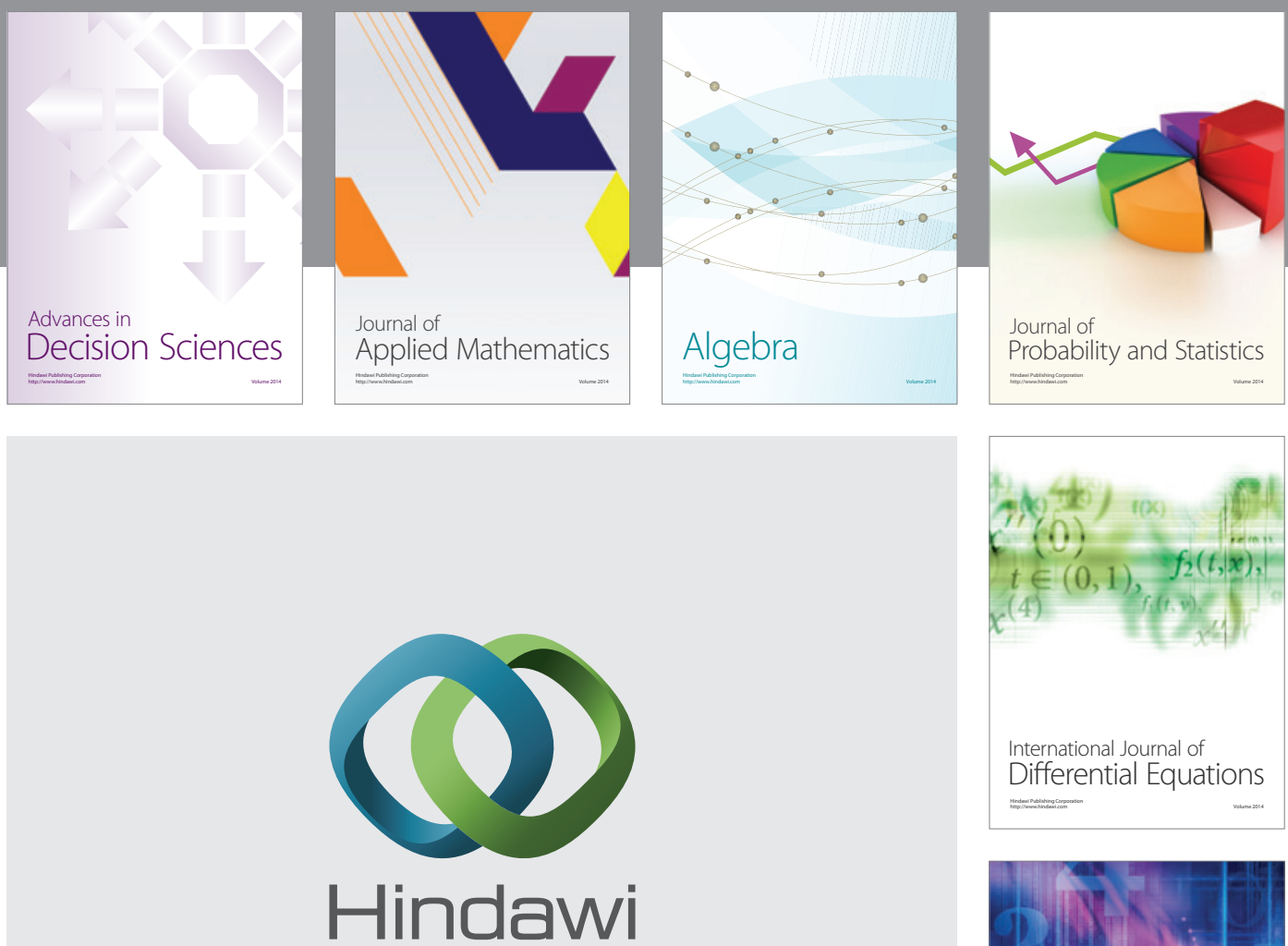

Submit your manuscripts at http://www.hindawi.com
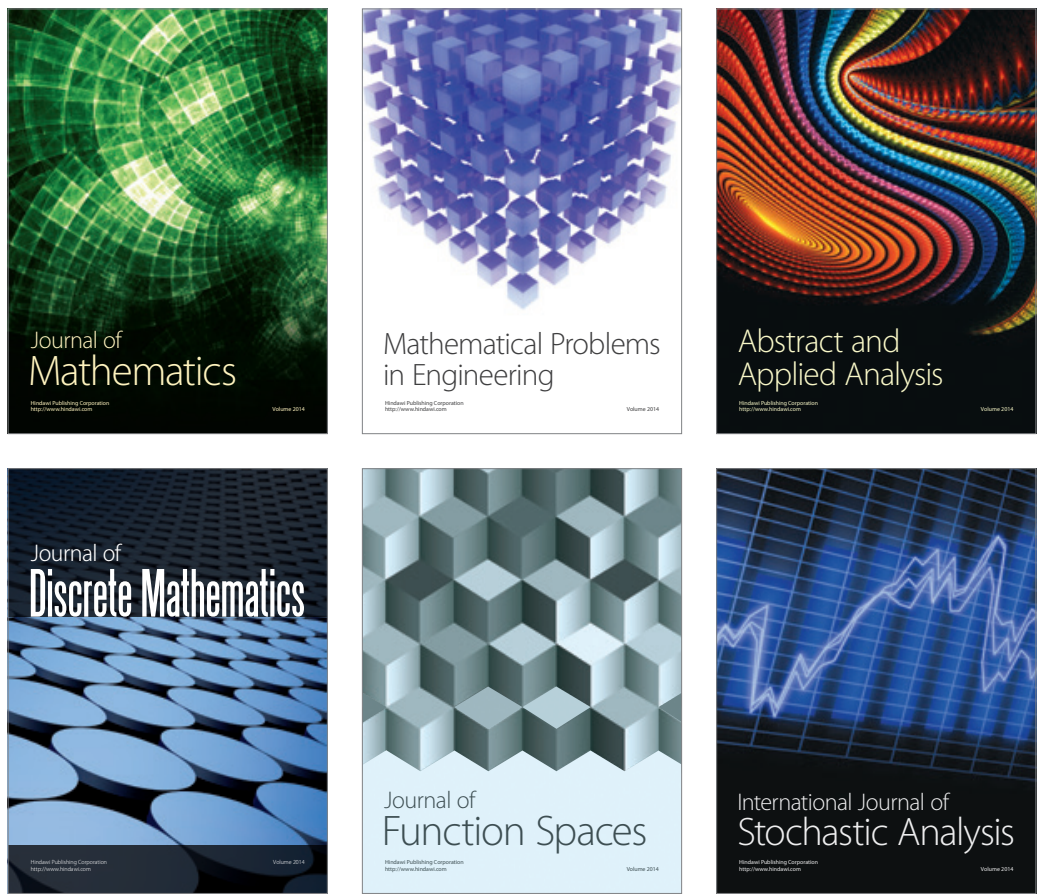

Journal of

Function Spaces

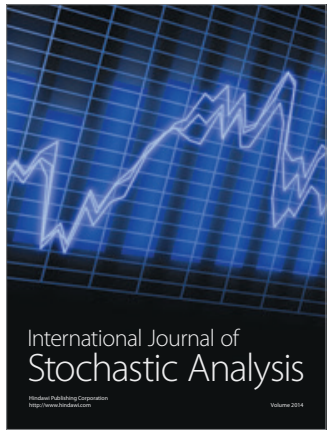

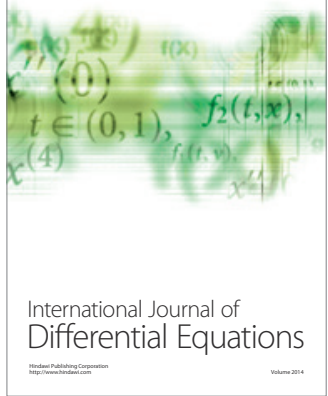
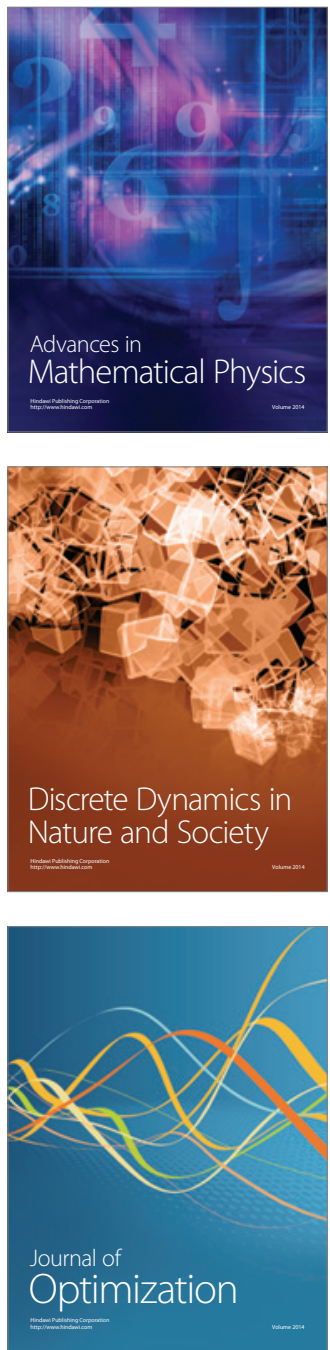